\title{
Antifungal therapy in patients with pulmonary Candida spp. colonization may have no beneficial effects
}

\author{
Simone Lindau', Manuel Nadermann ${ }^{1}$, Hanns Ackermann², Tobias Michael Bingold ${ }^{1}$, Christoph Stephan ${ }^{3}$, \\ Volkhard A. J. Kempf ${ }^{4}$, Pia Herzberger ${ }^{4}$, Andres Beiras-Fernandez ${ }^{5}$, Kai Zacharowski ${ }^{1}$ and Patrick Meybohm ${ }^{1 *}$
}

\begin{abstract}
Background: In critically ill patients, Candida spp. can often be identified in pulmonary samples. The impact of prompt antifungal therapy in these patients is unknown.

Methods: In this retrospective study, 500 adult patients with pulmonary Candida spp. colonization admitted to the intensive care unit (ICU) between 2010 and 2012 were included. The patients were analyzed according to whether or not they received antifungal therapy, which was administered at the discretion of the attending physician. Logistic regression analysis was performed to investigate the impact of antifungal therapy on hospital mortality and new onset of ventilator-associated pneumonia. In a stepwise backward elimination, the impact of age, cancer as an underlying disease, Simplified Acute Physiology Score (SAPS) II, and Sequential Organ Failure Assessment (SOFA) score were considered.
\end{abstract}

Results: After excluding 178 patients with multifocal Candida spp., isolated pulmonary Candida spp. colonization was found in 322 patients (cohort 1). Pre-existing pneumonia was found in 147/322 patients. Out of the remaining 175 patients (cohort 2), 44 patients received any antifungal therapy, and 131 were defined as the control group. Patients who received antifungal therapy had higher hospital mortality (50 vs. $30 \%, p=0.02$ ) and pneumonia rates (47.7 vs. $16.8 \% ; p<0.001$ ) than those who did not. In Cox regression analysis, antifungal therapy was not independently associated with favorable outcome (mortality: odds ratio 0.854 (95\% Cl 0.467-1.561); new pneumonia: 1.048 $(0.536-2.046))$, but SAPS II and SOFA score were significantly $(p<0.05)$ independent covariates for worse outcome.

Conclusions: In critically ill patients with pulmonary Candida spp. colonization, antifungal therapy may not have an impact on the incidence of new pneumonia or in-hospital mortality after adjustment for confounders.

Keywords: Candida spp. colonization, Antifungal therapy, Pneumonia

\section{Background}

Yeasts are part of the physiological flora of the oral mucosa and the intestine tract in about $40-65 \%$ of healthy adults [1]. In immunosuppressed patients, however, yeasts can cause severe infections [2]. The same may be true for non-neutropenic critically ill patients, where yeasts can infrequently be found after a prolonged interval of medical illness with complex modulation of the immune system. This particularly refers to critically

\footnotetext{
* Correspondence: patrick.meybohm@kgu.de

'Department of Anesthesiology, Intensive Care Medicine and Pain Therapy, University Hospital Frankfurt, Frankfurt, Germany

Full list of author information is available at the end of the article
}

ill patients with ongoing intravascular catheters, prolonged antibiotic therapy, chemotherapy, or long-term ventilation [3]. In clinical routine, Candida spp. can very often be identified in pulmonary samples taken from tracheal aspirates or bronchoalveolar lavage. However, the key question remains whether a simple colonization or an invasive Candida spp. infection exists. Meersseman et al. recently published that $57 \%$ of their deceased patients had findings of Candida albicans in the pulmonary secretion, but none of these patients had a "true" Candida spp. pneumonia [4]. For that reason, the European Society for Clinical Microbiology and Infectious Diseases (ESCMID) recommends that 
"Candida spp. isolation from respiratory secretions alone should never prompt treatment" [5].

In contrast, multifocal Candida spp. findings increase the risk of a systemic Candida spp. infection, and thereby increase risk for morbidity and mortality [6]. León et al. recently reported the Candida colonization index as a risk score for systemic Candida spp. infection including the status of (a) multifocal colonization, (b) surgery, (c) parenteral nutrition, and (d) severe sepsis [7].

Interestingly, Azoulay et al. found in a multicenter cohort of 800 patients that pulmonary Candida spp. colonization was significantly associated with an increased risk of nosocomial pneumonia and prolonged length of stay at the intensive care unit (ICU) [8]. Additionally, Hamet et al. reported in 300 critically ill patients with ventilator-associated pneumonia that pulmonary Candida spp. colonization was found in $56 \%$ of these patients and represents an independent risk factor for multidrugresistant bacterial super-infection associated with an increased risk of mortality [9].

In this respect, whether or not antifungal therapy should be initiated in critically ill patients with pulmonary Candida spp. colonization remains to be elucidated. In our retrospective study, we hypothesized that initiation of any antifungal therapy was associated with a reduced risk of new onset of ventilator-associated pneumonia and death compared to no antifungal therapy.

\section{Methods}

This is a retrospective study including 500 intensive care patients with at least one pulmonary finding of Candida spp. The patients were treated between 2010 and 2012 at the intensive care unit of the Department of Anesthesiology, Intensive Care Medicine and Pain Therapy, University Hospital Frankfurt, Germany. This study was approved by the ethical committee of the Faculty of Medicine, University Hospital Frankfurt (379/12). The ethic committee did not have any ethical concerns and confirmed that no patient consent form is needed as this study retrospectively analyzed routine data.

As a clinical routine, pulmonary samples were taken as tracheal aspirates tracheal aspirates for microbiological analyses twice a week and additionally when a new pulmonary infection was considered (tracheal aspirates or bronchoalveolar lavage). The samples were created in laboratory natively and after dilution quantitatively on nutrient agar. The results were considered positive in the presence of Candida spp. growth in the culture medium. The different Candida isolates were identified at species level. Serologic biomarkers for Candida spp. were not used routinely. Indication for antifungal treatment was based on an individual decision considering the underlying risk factors of the critically ill patient, respectively.
These factors particularly covered neutropenia, renal replacement therapy, long-term ventilation, or multifocal colonization.

\section{Endpoints}

Primary endpoints were in-hospital mortality or any new onset of ventilator-associated pneumonia. Ventilatorassociated pneumonia was defined as present (a) if the attending physician declared a new onset of a pulmonary infection and indicated broad-spectrum antibiotics or (b) if a new onset of pneumonia was defined according to the clinical and microbiological criteria of the CDC (Centers for Disease Control and Prevention) within the German Hospital Infection Surveillance System [10].

Secondary endpoints were length of stay at the intensive care unit, duration of ventilation based on the documented time of ventilation within the DRG coding, length of hospital stay, SAPS II (Simplified Acute Physiology Score), SOFA (Sequential Organ Failure Assessment) score at baseline and day 7 (without nervous system), and systemic inflammatory mediators at baseline and days 3, 7, and 14 including C-reactive protein, procalcitonin, leukocytes, thrombocytes, interleukin-6, and lipoprotein-binding protein. Also, we documented gender, primary discipline, cancer, any antibiotics and antifungal therapy, any pulmonary microbiology findings, and occurrence of sepsis and septic shock. Data collection was started as soon as the first positive microbiological finding with Candida spp. was reported (baseline). The observation time period for microbiological results and therapy was limited to a maximum of 28 days of ICU therapy.

\section{Sample size}

This study has a retrospective observational design and is exploratory in nature and undertaken for description. Therefore, no direct comparisons between different antifungal strategies and Candida spp. have been performed.

For sample size calculation, we took into account previous study results. Risk of ventilator-associated pneumonia was significantly increased in patients with Candida spp. colonization (24\% out of 214 patients vs. $19 \%$ [8] and $32 \%$ out of 181 patients vs. $23 \%$ [9]). Antifungal treatment reduced the risk of ventilator-associated pneumonia in 36 patients with Candida spp. colonization by $50 \%$ [11]. We estimated that about $25 \%$ of our total cohort $(n=500)$ with pulmonary Candida spp. colonization would have received any antifungal drug resulting in about 80 patients for the therapy group compared to 350 untreated patients (control group).

Based on the above-mentioned clinical studies, incidence of ventilator-associated pneumonia was assumed to be about $30 \%$ in untreated patients with positive Candida spp. colonization (control group). We assumed that antifungal therapy reduces the incidence of pneumonia by 
$50 \%$ with a level of significance of 0.05 and a power of $80 \%$ by a two-sided test.

\section{Statistics}

Metrically scaled variables were presented as mean \pm standard deviation (SD) or median (25\%; $75 \%$ quartile). The $p$ values were determined by using the two-sided Wilcoxon-Mann-Whitney test. Nominally scaled variables were presented in absolute terms and percentage, based on the type of group. The $p$ values were determined by using the Fisher-Yates test.

To investigate the impact of any antifungal therapy in pulmonary colonized patients on the incidence of pneumonia and on in-hospital mortality, we performed Kaplan-Meier analysis with Mantel-Cox logrank test for survival time and pneumonia-free time as the dependent variables. Additionally, we performed Cox regression analysis considering the impact of age, cancer as an underlying disease, SAPS II, and SOFA score (baseline values). The regression results are displayed as odds ratio (95\% confidence interval). The number of cases and these five predictor variables in Cox models stick to the rule of thumb, using around ten outcome events per predictor variable $[12,13]$. In a final step, we performed a competing risk analysis (Aalen-Johansen estimator and logrank test) for pneumonia and death as competing events.

All statistics were two-tailed. Statistically significance was considered with a $p<0.05$.
The data have been analyzed using IBM SPSS statistics 22 for Mac OS X and Bias 10.12.

\section{Results}

Between 2010 and 2012, approximately 6000 patients were admitted to our ICU, of whom we analyzed 500 patients with any pulmonary finding of Candida spp. In the first step, we excluded patients with multifocal Candida spp. findings $(n=178)$, as multifocal colonization increases risk of systemic Candida spp. infection and triggers antifungal therapy. Isolated pulmonary Candida spp. colonization was found in 322 patients (cohort 1), of whom 102 patients received any antifungal therapy. Preexisting pneumonia at baseline was found in $147 / 322$ patients. Out of the remaining 175 patients (cohort 2), 44 patients did receive (therapy group) and $131 \mathrm{did}$ not receive any antifungal therapy (control group; Fig. 1). To elucidate the role of antifungal therapy in high-risk patients of suspected candidemia, post hoc subgroup analysis was performed in patients with a permanent pacemaker placement (cohort 3).

\section{Cohort $1(n=322)$}

Baseline characteristics of patients with isolated pulmonary Candida spp. colonization including patients with pre-existing pneumonia are presented in Table 1 and Additional files 1 and 2. Most of the patients had cardiac surgery $(n=142)$. The patients with antifungal therapy were more critically ill with significantly $(p<0.001)$

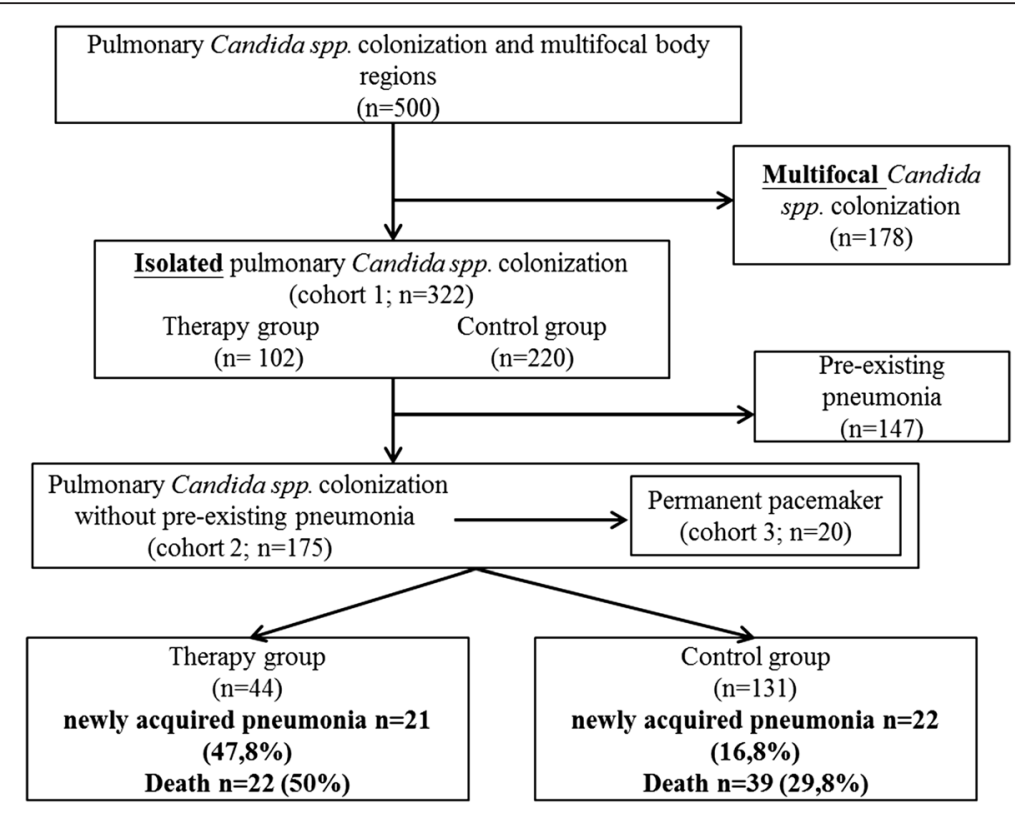

Fig. 1 Flow chart illustrating the recruitment process. 500 patients were eligible for this study. Cohort 1: 322 patients with isolated pulmonary Candida spp. colonization with or without pre-existing pneumonia after excluding 178 patients with multifocal colonization. Cohort 2: 175 patients with isolated pulmonary Candida spp. colonization without any pre-existing pneumonia (therapy group: $n=44$; control group: $n=131$ ) after excluding 147 patients who had a pre-existing pneumonia before enrollment 
higher SAPS II and SOFA scores, higher percentage of pre-existing pneumonia (56.9 vs. $40.5 \%$ ), higher use of carbapenems, quinolones, and glycopeptides, and higher incidence of Candida non-albicans species at baseline. Additional file 1 presents antimicrobial therapy. Antibiotics were given in $99 \%$ of the patients in the therapy group (vs. $84.1 \%$ ) 39 out of 102 patients (38.2\%) patients (38.2 \%) already received antifungal therapy at baseline, and in the rest of the therapy group, antifungal therapy was started in median after 4 days $(1 ; 6)$. The mean duration of antifungal therapy was $9(5 ; 15)$ days. Additional file 2 presents microbiological findings before enrollment. Most common Candida spp. were C. albicans (69.6 vs.

Table 1 Baseline characteristics of patients with isolated pulmonary Candida spp. colonization (cohort 1)

\begin{tabular}{|c|c|c|c|}
\hline & $\begin{array}{l}\text { Antifungal therapy } \\
(n=102)\end{array}$ & $\begin{array}{l}\text { No antifungal therapy } \\
(n=220)\end{array}$ & $p$ value \\
\hline Age (years) & $69(57 ; 76)$ & $70(58 / 78)$ & 0.354 \\
\hline Sex male, $n(\%)$ & $83(81.4)$ & 162 (73.6) & 0.16 \\
\hline \multicolumn{4}{|l|}{ Discipline, $n(\%)$} \\
\hline $\begin{array}{l}\text { Thoracic and } \\
\text { cardiovascular } \\
\text { surgery }\end{array}$ & $52(50.1)$ & $90(40.9)$ & 0.093 \\
\hline $\begin{array}{l}\text { General and } \\
\text { visceral surgery }\end{array}$ & $12(11.8)$ & $33(15.0)$ & 0.493 \\
\hline Trauma surgery & $9(8.8)$ & $29(13.2)$ & 0.353 \\
\hline Vascular surgery & $12(11.8)$ & $25(11.4)$ & 1 \\
\hline Urology & $3(2.9)$ & $1(0.5)$ & 0.096 \\
\hline Otolaryngology & $0(0)$ & $5(2.3)$ & 0.183 \\
\hline Pneumology & $3(2.9)$ & $4(1.8)$ & 0.683 \\
\hline $\begin{array}{l}\text { Oral and } \\
\text { maxillofacial } \\
\text { surgery }\end{array}$ & $0(0)$ & $4(1.8)$ & 0.311 \\
\hline Neurosurgery & $3(2.9)$ & $20(9.1)$ & 0.061 \\
\hline Others & $8(7.8)$ & $9(4.1)$ & 0.184 \\
\hline Cancer, n (\%) & 14 (13.7) & $42(19.1)$ & 0.271 \\
\hline $\begin{array}{l}\text { Permanent } \\
\text { pacemaker, } n(\%)\end{array}$ & $15(14.7)$ & $17(7.7)$ & 0.071 \\
\hline SAPS $\|$ & $47(39 ; 55)$ & $40(30 ; 48)$ & $<0.001$ \\
\hline SOFA score & $7(5 ; 10)$ & $4.5(2 ; 8)$ & $<0.001$ \\
\hline Lung & $2(1 ; 2)$ & $1(1 ; 2)$ & 0.437 \\
\hline Thrombocytes & $1(0 ; 2)$ & $0(0 ; 2)$ & 0.015 \\
\hline Liver & $0(0 ; 2)$ & $0(0 ; 0)$ & $<0.001$ \\
\hline Cardiovascular & $3(0 ; 4)$ & $0(0 ; 3)$ & 0.001 \\
\hline Renal & $1(0 ; 2)$ & $0(0 ; 2)$ & 0.017 \\
\hline $\begin{array}{l}\text { Pre-existing } \\
\text { pneumonia before } \\
\text { enrollment, } n(\%)\end{array}$ & $58(56.9)$ & $89(40.5)$ & 0.008 \\
\hline
\end{tabular}

Data are presented as median (25\%; $75 \%$ quartile) SAPS II Simplified Acute Physiology Score II, SOFA Sequential Organ Failure Assessment
$81.8 \%)$. Interestingly, significantly more therapy patients had any pulmonary bacterial findings at baseline ( 25 vs. $43 \%, p=0.002$ ).

Outcome parameters are displayed in Table 2. In the therapy group, SOFA score on day 7 was higher $(p<0.005), 47.7 \%$ of patients developed new pneumonia (vs. $16.8 \%$ ), durations of mechanical ventilation, hospital and ICU stay were longer $(p<0.001)$, more patients developed sepsis $(p<0.002)$ or septic shock $(p<0.006)$, and higher levels of pro-inflammatory mediators were found $(p<0.05$; Additional file 3$)$. Microbiological findings and therapy are summarized in Additional files 4 and 5.

Using the Kaplan-Meier estimate, survival curves did not show any significant difference between the groups ( $p>0.05$; Fig. 2a). Cox regression analysis revealed SAPS II (1.028 (1.008-1.048); $p=0.005)$, SOFA score (1.094 (1.030-1.163); $p=0.004)$, and age (1.020 (1.004-1.037); $p=0.016)$ as independent covariates for mortality, but antifungal therapy had no independent impact on chance of survival (1.198 (0.810-1.771); $p=0.366$; Additional file 6).

\section{Cohort $2(n=175)$}

Focusing on patients with isolated pulmonary Candida spp. colonization without pre-existing pneumonia, baseline characteristics are presented in Table 3. SAPS II $(p<0.001)$ and SOFA score were significantly higher in the therapy group $(p<0.003)$. Additional file 7 presents microbiological findings and therapy. In 17 of 44 patients $(38.6 \%)$, preemptive antifungal therapy was already administered at baseline. Outcome parameters of the cohort 2 are presented in Table 4 . In the therapy group, more patients became septic ( 40.9 vs. $20.6 \%$ ), had longer hospital $(p<0.005)$ and ICU $(p<0.001)$ stays, a higher incidence of newly acquired pneumonia $(p<0.001)$, and died more often (50 vs. $29.8 \% ; p=0.02$ ). During the observation period, $52.3 \%$ had any evidence of pulmonary bacterial colonization/infection. Gram-negative bacteria were the most common co-infection (Additional file 8).

Kaplan-Meier estimate did not show a significant difference in the survival curves between the therapy and control group ( $p>0.05$; Fig. 2b). Using the Cox regression analysis, antifungal therapy had no significant independent impact on survival (0.855 (0.469-1.558), $p=0.608)$, but SAPS II (1.027 (1.003-1.052), $p=0.025)$ and SOFA score (1.096 (1.004-1.198), $p=0.041)$ were independently associated with higher risk of death (Additional file 9). The time until new hospital-acquired pneumonia was significantly shorter in the therapy group $(p<0.001$; Fig. 3$)$, but again, antifungal therapy had no significant impact using Cox regression analysis (1.034 (0.546-1.957), $p=0.919$; Additional file 10). 
Table 2 Outcome characteristics of patients with isolated pulmonary Candida spp. colonization (cohort 1)

\begin{tabular}{|c|c|c|c|}
\hline & $\begin{array}{l}\text { Antifungal } \\
\text { therapy }(n=102)\end{array}$ & $\begin{array}{l}\text { No antifungal } \\
\text { therapy }(n=220)\end{array}$ & $p$ value \\
\hline SOFA score (day 7) & $6(3 ; 9)$ & $3(2 ; 7)$ & 0.003 \\
\hline Lung & $1(1 ; 2)$ & $1(1 ; 2)$ & 0.034 \\
\hline Thrombocytes & $1(0 ; 2)$ & $0(0 ; 2)$ & 0.236 \\
\hline Liver & $0(0 ; 1)$ & $0(0 ; 0)$ & 0.001 \\
\hline Cardiovascular & $0(0 ; 3)$ & $0(0 ; 3)$ & 0.033 \\
\hline Renal & $1(0 ; 2)$ & $1(0 ; 2)$ & 0.159 \\
\hline New pneumonia, $n(\%)$ & $21(20.6)$ & $22(10)$ & 0.013 \\
\hline \multicolumn{4}{|l|}{ Sepsis, n (\%) } \\
\hline Sepsis/severe sepsis & $27(26.5)$ & $26(11.8)$ & 0.002 \\
\hline Septic shock & $29(28.4)$ & $32(14.5)$ & 0.006 \\
\hline $\begin{array}{l}\text { Duration of mechanical } \\
\text { ventilation }(\mathrm{h})\end{array}$ & $362(199 ; 599)$ & $160(67 ; 284)$ & $<0.001$ \\
\hline Hospital stay (days) & $31(17 ; 53)$ & $21(12 ; 35)$ & $<0.001$ \\
\hline ICU stay (days) & $20(11 ; 29)$ & $8(4 ; 14)$ & $<0.001$ \\
\hline In-hospital mortality, n (\%) & $53(52.0)$ & $76(34.5)$ & 0.003 \\
\hline \multicolumn{4}{|l|}{ Discharge from hospital, $n$ (\%) } \\
\hline Other hospital & $24(23.5)$ & $47(21.4)$ & 0.667 \\
\hline Rehabilitation clinic & 19 (18.6) & $42(19.1)$ & 1 \\
\hline Home & $6(5.9)$ & $49(22.3)$ & $<0.001$ \\
\hline Others & $0(0)$ & $6(2.7)$ & 0.032 \\
\hline
\end{tabular}

Data are presented as median ( $25 \% ; 75 \%$ quartile)

SOFA Sequential Organ Failure Assessment, ICU intensive care unit
In the final step, competing risk analysis with the Aalen-Johansen estimator for pneumonia and death as the competing events was performed. Again, the time until new event was significantly shorter in the therapy group $(p<0.001)$ (not shown).

\section{Cohort $3(n=20)$}

In case that blood culture shows false negative candidemia, we defined a subgroup of high-risk patients of suspected candidemia who had a permanent pacemaker placement (cohort 3). The baseline characteristics are presented in Table 5. SAPS II and SOFA score did not significantly differ between the groups. Mortality rate did not differ significantly (therapy group 4/8 (50 \%) vs. control group 6/12 (50 \%)). Using the Cox regression analysis, antifungal therapy had no significant independent impact on survival $(1.693(0.396-7.230), p=0.477)$. The rate of new hospital-acquired pneumonia did not differ significantly (therapy group 2/8 (25 \%) vs. control group 3/12 (25\%)). Antifungal therapy had no significant impact using Cox regression analysis (0.448 (0.11-18.71), $p=0.673)$.

\section{Discussion}

Candida is frequently found in pulmonary secretions of patients in ICU known as Candida spp. colonization, if clinical and pathological evidence of invasive candidiasis is absent. In this respect, Candida spp. colonization may not play an important pathogenic role in the context of ventilator-associated pneumonia, at least in immunocompetent patients [4]. Consequently, the European Society for Clinical Microbiology and Infectious Diseases (ESCMID) do not recommend a systemic antifungal

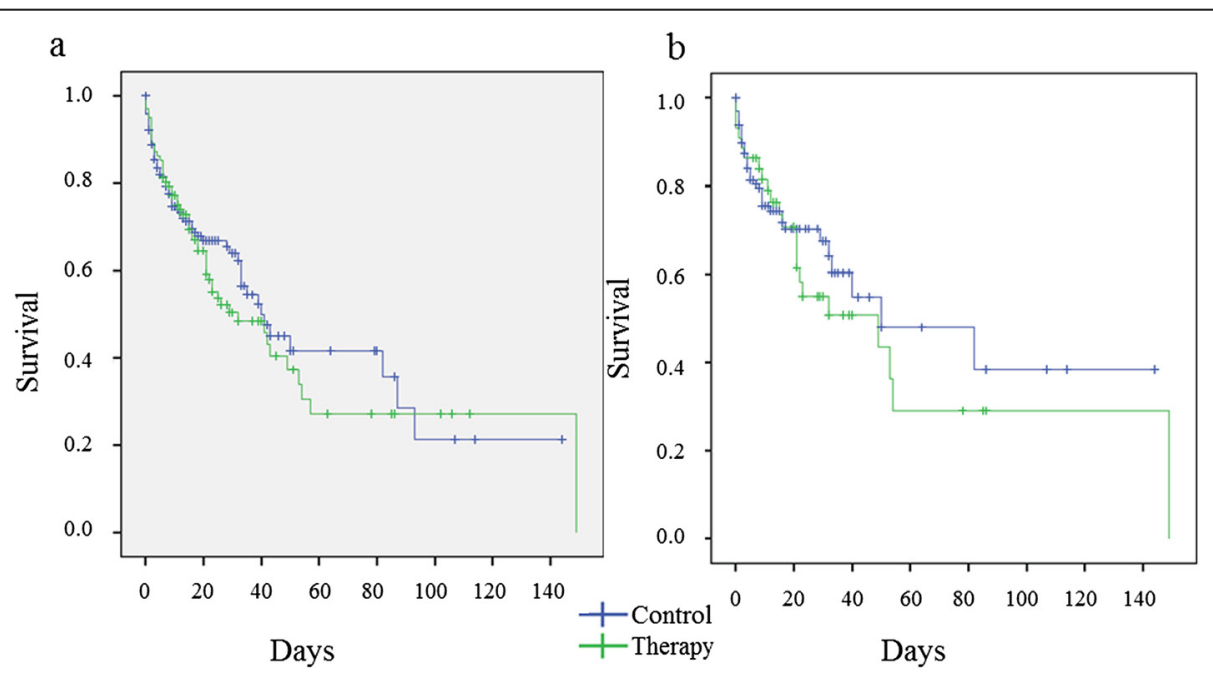

Fig. 2 Kaplan-Meier analysis for survival time. a Cohort 1: patients with isolated pulmonary Candida spp. colonization $(n=322)$ including patients with pre-existing pneumonia; Mantel-Cox logrank test: $p>0.05$. b Cohort 2: patients with isolated pulmonary Candida spp. colonization $(n=175)$ excluding patients with pre-existing pneumonia before enrollment; Mantel-Cox Logrank test: $p>0.05$. Therapy group (green line) vs. control group (blue line) 
Table 3 Baseline characteristics of patients with isolated pulmonary Candida spp. colonization without pre-existing pneumonia (cohort 2)

\begin{tabular}{llll}
\hline & $\begin{array}{l}\text { Antifungal } \\
\text { therapy }(n=44)\end{array}$ & $\begin{array}{l}\text { No antifungal } \\
\text { therapy }(n=131)\end{array}$ & $p$ value \\
\hline Age (years) & $70(59 ; 76)$ & $71(56 ; 79)$ & 0.57 \\
Sex male, $n(\%)$ & $38(86.4)$ & $94(71.8)$ & 0.07 \\
Cancer, $n(\%)$ & $5(11.4)$ & $24(18.3)$ & 0.35 \\
Permanent & $8(18.2)$ & $12(9.1)$ & 0.17 \\
pacemaker, $n(\%)$ & & & $<0.001$ \\
SAPS II & $48(37 ; 55)$ & $37(26 ; 47)$ & 0.003 \\
SOFA score & $7(4 ; 10)$ & $4(2 ; 8)$ & \\
\hline
\end{tabular}

Data are presented as median (25\%; $75 \%$ quartile)

SAPS I/ Simplified Acute Physiology Score II, SOFA Sequential Organ

Failure Assessment

therapy based on isolated findings of Candida spp. in pulmonary secretion [5].

On the other hand, pulmonary Candida spp. colonization has been shown to be significantly associated with an increased risk of nosocomial pneumonia [8]. Additionally, pulmonary Candida spp. colonization is suggested to be an independent risk factor for multidrug-resistant bacterial super-infection associated with an increased risk of mortality [9]. In this respect, any therapeutic intervention to attenuate fungal pathogens might be an interesting option, at least in theory. In clinical routine, the individual decision to treat or not to treat is very difficult, and patients often receive an antifungal drug at the discretion of the individual attending physician irrespective of the guidelines.

Very recently, Albert et al. included 60 patients into a randomized trial and analyzed 29 patients within an observational study, whether antifungal therapy in

Table 4 Outcome characteristics of patients with isolated pulmonary Candida spp. colonization without pre-existing pneumonia (cohort 2)

\begin{tabular}{llll}
\hline & $\begin{array}{l}\text { Antifungal } \\
\text { therapy }(n=44)\end{array}$ & $\begin{array}{l}\text { No antifungal } \\
\text { therapy }(n=131)\end{array}$ & $p$ value \\
\hline SOFA score (day 7$)$ & $7(4 ; 10)$ & $3(1 ; 6)$ & 0.003 \\
$\begin{array}{l}\text { Newly acquired } \\
\text { pneumonia, } n(\%)\end{array}$ & $21(47.7)$ & $22(16.8)$ & $<0.001$ \\
$\begin{array}{l}\text { Sepsis/severe sepsis, } \\
n \text { (\%) }\end{array}$ & $18(40.9)$ & $27(20.6)$ & 0.01 \\
$\begin{array}{l}\text { Mechanical } \\
\text { ventilation (h) }\end{array}$ & $162(140 ; 547)$ & $152(59 ; 242)$ & $<0.001$ \\
$\begin{array}{l}\text { Hospital stay (days) } \\
\text { ICU stay (days) }\end{array}$ & $31(15 ; 53)$ & $20(10 ; 34)$ & 0.004 \\
$\begin{array}{l}\text { In-hospital mortality, } \\
n(\%)\end{array}$ & $22(50)$ & $7(4 ; 12)$ & $<0.001$ \\
\hline Data are presented as median $(25 \% ; 75 \%)$ & $39(29.8)$ & 0.02 \\
\hline
\end{tabular}

Data are presented as median ( $25 \%$; $75 \%$ ) quartile SOFA Sequential Organ Failure Assessment critically ill patients with a clinical suspicion of ventilatorassociated pneumonia with positive airway secretion specimens for Candida spp. might be beneficial [12]. Markers of inflammation and all clinical outcomes were comparable between placebo and antifungal treatment group at baseline and over time. These authors concluded that this study did not provide evidence to support a larger trial examining the efficacy of empiric antifungal treatment in patients with a clinical suspicion of ventilator-associated pneumonia and Candida spp. in the tracheal secretions.

The main results of our retrospective study including 322 critically ill patients are in line with these recent findings. Using Cox regression analysis to account for confounders, any antifungal treatment did not had a beneficial effect on the incidence of new pneumonia or in-hospital mortality in a cohort of heterogenic intensive care patients with isolated pulmonary Candida spp. colonization. Not surprisingly, patients that received antifungal therapy were more critically ill as evident from higher SAPS II and SOFA score and had a higher degree of systemic inflammation. In this respect, these patients showed prolonged duration of mechanical ventilation, ICU, and hospital length of stay.

Consistently, our Cox regression analysis revealed SAPS II, SOFA score, and age of patient as independent variables for a higher risk of in-hospital mortality. These results are not surprising, as SAPS II "...provides an estimate of the risk of death without having to specify a primary diagnosis" [14], and the SOFA score reasonably describes organ dysfunction in critically ill patients [15]. Nfor et al. evaluated a direct relationship between the total SOFA score at admission and ICU mortality [16]. Moreno et al. showed that ICU mortality was more strongly associated with 'delta' SOFA score, which is defined as the total maximum SOFA minus the SOFA score at admission [17].

In a subgroup of patients (cohort 2), we focused on the time until new pneumonia. If any antifungal therapy is suggested to be beneficial, then free time to any new pneumonia should reasonably be longer, at least in theory. Surprisingly, our results showed contradictory effects, and time until new pneumonia was significantly shorter in patients that received antifungal therapy during ICU stay. This is in line with our findings that patients with antifungal therapy were more critically ill with significantly higher incidence of new pneumonia, higher SAPS II and SOFA score, higher incidence of sepsis, and longer duration of hospital and ICU stays. Thus, severity of critical illness may have a large bias on outcome variables. To account for these confounders, we considered a couple of known covariates for pneumonia in our Cox regression analysis. Nevertheless, antifungal therapy may not have an independent effect on time to new 


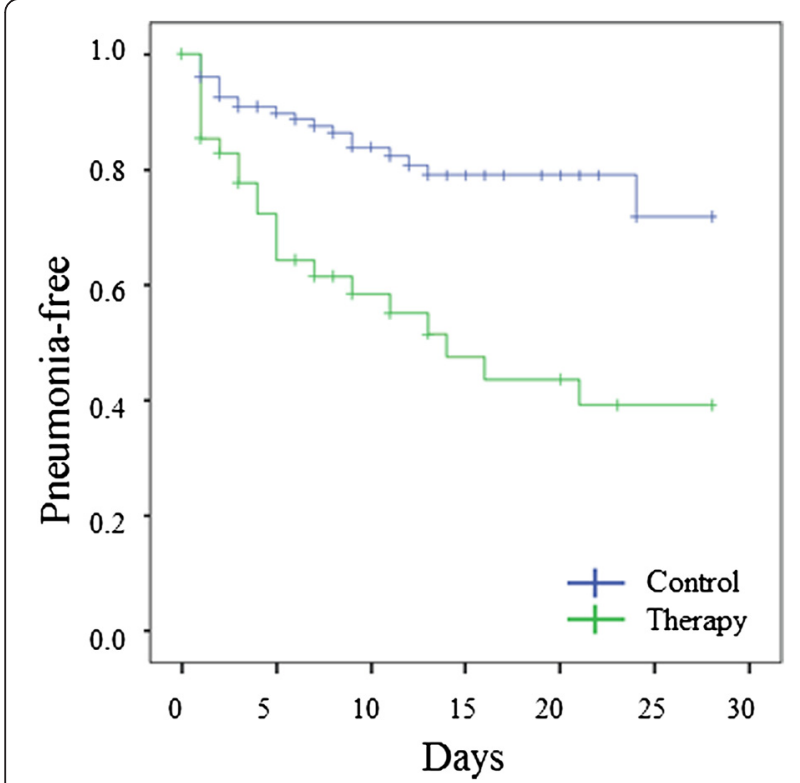

Fig. 3 Kaplan-Meier analysis for time until new ventilator-associated pneumonia. Cohort 2: Patients with isolated pulmonary Candida spp. colonization without any pre-existing pneumonia before enrollment $(n=175)$; Mantel-Cox logrank test: $p<0.001$. Therapy group (green line) vs. control group (blue line)

pneumonia or survival chance. There are three additional interesting observations: (a) most of the patients developed new pneumonia within the first 10 days after microbiological detection of yeasts, (b) preemptive antifungal therapy has been observed in $38 \%$ of the patients, and (c) median time to initiate antifungal therapy was 4 days in non-preemptive-treated patients. Under these circumstances, it remains speculative if a faster initiation of antifungal therapy at the first day of microbiological result or even a more preemptive antifungal strategy in critically ill patients would otherwise prevent any yeasts and would result in a better outcome. Nevertheless, these factors are of minor importance regarding the key question of this analysis-whether or not to

Table 5 Baseline characteristics of high-risk patients with permanent pacemaker and isolated pulmonary Candida spp. colonization without pre-existing pneumonia (cohort 3)

\begin{tabular}{llll}
\hline & $\begin{array}{l}\text { Antifungal therapy } \\
(n=8)\end{array}$ & $\begin{array}{l}\text { No antifungal therapy } \\
(n=12)\end{array}$ & $p$ value \\
\hline Age (years) & $73(65 ; 79)$ & $74(69 ; 83)$ & 0.69 \\
Cancer, $n(\%)$ & $0(0)$ & $4(30)$ & 0.12 \\
SAPS II & $50(46 ; 56)$ & $34(29 ; 58)$ & 0.09 \\
SOFA score & $10(5 ; 11)$ & $4(3 ; 7)$ & 0.08 \\
\hline
\end{tabular}

Data are presented as median (25\%; $75 \%$ quartile) SAPS II Simplified Acute Physiology Score II, SOFA Sequential Organ Failure Assessment initiate antifungal therapy in critically ill patients with Candida spp. colonization.

Our results also suggest that the absence of any beneficial effect after antifungal therapy is irrespective of any pre-existing pneumonia. Thus, even in higher risk critically ill patients with pre-existing ventilator-associated pneumonia (cohort 1), the presence of Candida spp. in the lung could be interpreted as harmless colonization rather than representing a true infection requiring any treatment [18]. Very recently, Blot et al. found several risk factors for mortality in patients with ventilator-associated pneumonia, e.g., diabetes and septic shock [19], but similarly Candida spp. colonization had no significant impact in this study.

Thus, our results support the recent ESCMID recommendations, not to initiate antifungal therapy in patients with isolated pulmonary Candida spp. colonization irrespective of the severity of critical illness.

Importantly, blood culture may show false negative results of candidemia among high-risk patients with central venous catheter or permanent pacemaker placement due to relatively slow growth of Candida spp. in the blood culture. To elucidate the role of antifungal therapy in these high-risk patients, we performed a subgroup analysis of 20 patients with implanted pacemaker and isolated pulmonary Candida spp. colonization without pre-existing pneumonia. Again, antifungal therapy was not independently associated with a favorable outcome.

Our study has the following limitations. Firstly, this study has a retrospective and explorative design, and a large prospective randomized controlled study might be needed to provide more evidence whether or not a fungal therapy may be beneficial in patients with isolated pulmonary Candida spp. colonization. Secondly, we did not consider additional risk factors for ventilator-associated pneumonia as covariates, e.g., chronic obstructive pulmonary disease [20], the use of broad-spectrum antibiotics in the preceding days [21], diabetes mellitus [22], and the use of proton pump inhibitors [23], as these parameters would not be validly available using a retrospective design in our hospital. Finally, it is well known that different Candida spp. (albicans, krusei, etc.) have different pathogenicity and sensibilities to drugs, but we did not perform subgroup analyses regarding different types of Candida spp. or kinds of antifungal drugs, as the sample size is reasonable small.

\section{Conclusions}

Our study suggests that antifungal therapy may not have an impact on the incidence of new pneumonia or inhospital mortality after adjustment for confounders in critically ill patients with isolated pulmonary Candida spp. 


\section{Additional files}

Additional file 1: Baseline microbiological therapy in patients with isolated pulmonary Candida spp. colonization (cohort 1). Different antifungal drugs and antibiotics that were given at baseline are shown.

Additional file 2: Baseline pulmonary microbiological findings in patients with isolated pulmonary Candida spp. colonization (cohort 1).| Candida spp., Aspergillus and any bacterial findings at baseline are shown.

Additional file 3: Inflammatory mediators in patients with isolated pulmonary Candida spp. colonization (cohort 1). Different inflammatory mediators are shown on day 1, 3, 7 and 14 .

Additional file 4: Microbiological therapy in patients with isolated pulmonary Candida spp. colonization during observation period (cohort 1). Different antifungal drugs and antibiotics that were given during observation period are shown.

Additional file 5: Pulmonary microbiological findings in patients with isolated pulmonary Candida spp. colonization during observation period (cohort 1). Candida spp., Aspergillus and any bacterial findings during observation period are shown.

Additional file 6: Patients with isolated pulmonary Candida spp. colonization-stepwise backwards elimination for survival time (cohort 1). Cox regression analysis for independent impact on survival was performed for potential co-variable (Therapy, SAPS II, SOFA score, Age and Cancer).

Additional file 7: Baseline pulmonary microbiological findings and therapy in patients with isolated pulmonary Candida spp. colonization without pre-existing pneumonia (cohort 2). Different antifungal drugs and antibiotics that were given and Candida spp., Aspergillus and any bacterial findings at baseline are shown.

Additional file 8: Pulmonary microbiological findings and therapy in patients with isolated pulmonary Candida spp. colonization without pre-existing pneumonia during observation period (cohort 2). Different antifungal drugs and antibiotics that were given and Candida spp., Aspergillus and any bacterial findings during observation period are shown.

Additional file 9: Patients with isolated pulmonary Candida spp. colonization without pre-existing pneumonia-stepwise backwards elimination for survival time (cohort 2). Cox regression analysis for independent impact on survival was performed for potential co-variable (Therapy, SAPS II, SOFA score, Age and Cancer).

Additional file 10: Patients with isolated pulmonary Candida spp. colonization without pre-existing pneumonia-stepwise backwards elimination for pneumonia-free time (cohort 2). Cox regression analysis for independent impact on newly aquired pneumonia was performed for potential co-variable (Therapy, SAPS II, SOFA score, Age and Cancer).

\section{Abbreviations}

ESCMID: European Society for Clinical Microbiology and Infectious Diseases; ICU: intensive care unit; MDR: multidrug resistance; SAPS: Simplified Acute Physiology Score; SOFA: Sequential Organ Failure Assessment.

\section{Competing interests}

The authors declare that they have no competing interests.

\section{Authors' contributions}

SL, MN, and PM have made substantial contributions to the conception and design, acquisition of the data, analysis and interpretation of the data. VK and $\mathrm{PH}$ carried out microbiological analyses. HA participated in the design of the study and performed the statistical analysis. TB and CS conceived of the study, participated in its design and coordination, and helped to draft the manuscript. $A B F$ and $K Z$ have been involved in drafting the manuscript and revising it critically for important intellectual content. $K Z$ and $P M$ have given final approval of the version to be published. All authors read and approved the final manuscript.

\section{Acknowledgement}

The authors thank the staff of the ICU and the biological laboratory for their support that made this project possible.

\section{Author details}

'Department of Anesthesiology, Intensive Care Medicine and Pain Therapy, University Hospital Frankfurt, Frankfurt, Germany. ${ }^{2}$ Institute of Biostatistics and Mathematical Modelling, University Hospital Frankfurt, Frankfurt, Germany.

${ }^{3}$ Department of Medicine II: Infectiology, University Hospital Frankfurt, Frankfurt, Germany. ${ }^{4}$ Institute for Medical Microbiology and Infection Control, University Hospital Frankfurt, Frankfurt, Germany. ${ }^{5}$ Department of Thoracic and Cardiovascular Surgery, University Hospital Frankfurt, Frankfurt, Germany.

Received: 11 March 2015 Accepted: 24 June 2015

Published online: 03 July 2015

\section{References}

1. Zegarelli DJ. Fungal infections of the oral cavity. Otolaryngol Clin North Am. 1993:26:1069-89.

2. Dreizen S, Keating MJ, Beran M. Orofacial fungal infections. Nine pathogens that may invade during chemotherapy. Postgrad Med. 1992;91:349-50. 353-4, 357-60 passim.

3. Lichtenstern C, Swoboda S, Hirschburger M, Domann E, Hoppe-Tichy T, Winkler $M$, et al. Update: invasive Pilzinfektionen: Diagnose und Therapie in der operativen Intensivmedizin. Anaesthesist. 2010;59:30-52.

4. Meersseman W, Lagrou K, Spriet I, Maertens J, Verbeken E, Peetermans WE, et al. Significance of the isolation of Candida species from airway samples in critically ill patients: a prospective, autopsy study. Intensive Care Med. 2009;35:1526-31

5. Cornely OA, Bassetti M, Calandra T, Garbino J, Kullberg BJ, Lortholary O, et al. ESCMID* guideline for the diagnosis and management of Candida diseases 2012: non-neutropenic adult patients. Clin Microbiol Infect. 2012;18 Suppl 7:19-37.

6. Playford EG, Lipman J, Kabir M, McBryde ES, Nimmo GR, Lau A, et al. Assessment of clinical risk predictive rules for invasive candidiasis in a prospective multicentre cohort of ICU patients. Intensive Care Med. 2009;35:2141-5.

7. León C, Ruiz-Santana S, Saavedra P, Galván B, Blanco A, Castro C, et al. Usefulness of the "Candida score" for discriminating between Candida colonization and invasive candidiasis in non-neutropenic critically ill patients: a prospective multicenter study. Crit Care Med. 2009;37:1624-33.

8. Azoulay E, Timsit J, Tafflet M, de Lassence A, Darmon M, Zahar J, et al. Candida colonization of the respiratory tract and subsequent pseudomonas ventilator-associated pneumonia. Chest. 2006;129:110-7.

9. Hamet M, Pavon A, Dalle F, Pechinot A, Prin S, Quenot J, et al. Candida spp. airway colonization could promote antibiotic-resistant bacteria selection in patients with suspected ventilator-associated pneumonia. Intensive Care Med. 2012;38:1272-9.

10. Nationales Referenzzentrum für Surveillance von nosokomialen Infektionen Definition nosokomialer Infektionen (CDC-Definitionen); 2011. http:// www.nrz-hygiene.de/surveillance/kiss/cdc-definitionen/ (accessed 09. August 2014).

11. Nseir S, Jozefowicz E, Cavestri B, Sendid B, Di Pompeo C, Dewavrin F, et al. Impact of antifungal treatment on Candida-Pseudomonas interaction: a preliminary retrospective case-control study. Intensive Care Med. 2007;33:137-42.

12. Vittinghoff $E, M c C u l l o c h ~ C E$. Relaxing the rule of ten events per variable in logistic and Cox regression. Am J Epidemiol. 2007;165:710-8.

13. Herrmann E., M. Ahmed, M. Shahrashoub, A. Orth, H. Ackermann. Assessing approximative algorithms for calculating Spearman's rank correlation coefficient for moderate sample size in the case of ties. DAGStat, Statistics under one Umbrella, Dortmund; Germany: 2010.

14. Le Gall J. A new simplified acute physiology score (SAPS II) based on a European/North American multicenter study. JAMA. 1993;270:2957.

15. Vincent JL, de Mendonça A, Cantraine F, Moreno R, Takala J, Suter PM, et al. Use of the SOFA score to assess the incidence of organ dysfunction/failure in intensive care units: results of a multicenter, prospective study. Working group on "sepsis-related problems" of the European Society of Intensive Care Medicine. Crit Care Med. 1998;26:1793-800.

16. Nfor TK, Walsh TS, Prescott RJ. The impact of organ failures and their relationship with outcome in intensive care: analysis of a prospective multicentre database of adult admissions. Anaesthesia. 2006;61:731-8. 
17. Moreno R, Vincent IL, Matos R, Mendonça A, Cantraine F, Thijs L, et al. The use of maximum SOFA score to quantify organ dysfunction/failure in intensive care. Results of a prospective, multicentre study. Working group on sepsis related problems of the ESICM. Intensive Care Med. 1999;25:686-96.

18. Albert M, Williamson D, Muscedere J, Lauzier F, Rotstein C, Kanji S, et al. Candida in the respiratory tract secretions of critically ill patients and the impact of antifungal treatment: a randomized placebo controlled pilot trial (CANTREAT study). Intensive Care Med. 2014;40(9):1313-22.

19. Blot S, Koulenti D, Dimopoulos G, Martin C, Komnos A, Krueger WA, et al. Prevalence, risk factors, and mortality for ventilator-associated pneumonia in middle-aged, old, and very old critically ill patients*. Crit Care Med. 2014;42:601-9.

20. Rouzé A, Cottereau A, Nseir S. Chronic obstructive pulmonary disease and the risk for ventilator-associated pneumonia. Curr Opin Crit Care. 2014;20(5):525-31.

21. Ranjan N, Chaudhary U, Chaudhry D, Ranjan KP. Ventilator-associated pneumonia in a tertiary care intensive care unit: analysis of incidence, risk factors and mortality. Indian J Crit Care Med. 2014;18:200-4.

22. Benfield T, Jensen JS, Nordestgaard BG. Influence of diabetes and hyperglycaemia on infectious disease hospitalisation and outcome. Diabetologia. 2007;50:549-54.

23. Eom C, Jeon CY, Lim J, Cho E, Park SM, Lee K. Use of acid-suppressive drugs and risk of pneumonia: a systematic review and meta-analysis. CMAJ. 2011;183:310-9.

\section{Submit your next manuscript to BioMed Central and take full advantage of:}

- Convenient online submission

- Thorough peer review

- No space constraints or color figure charges

- Immediate publication on acceptance

- Inclusion in PubMed, CAS, Scopus and Google Scholar

- Research which is freely available for redistribution 